\title{
Modern-day prostate cancer is not meaningfully associated with lower urinary tract symptoms: Analysis of a propensity score- matched cohort
}

\author{
Amar Bhindi, MSc, ${ }^{, 1}$ Bimal Bhindi, MD; ${ }^{2}$ Girish S. Kulkarni, MD;, Robert J. Hamilton, MD, ${ }^{2}$ Ants Toi, MD; ${ }^{3}$ \\ Theodorus H. van der Kwast, MD,; Andrew Evans, MD,; Alexandre R. Zlotta, MD,2 Antonio Finelli, MD; \\ Neil E. Fleshner, $M D^{2}$
}

'Department of Medicine, McGill University, Montreal, QC, Canada; ${ }^{2}$ Division of Urology, Department of Surgery, University Health Network, University of Toronto, Toronto, ON, Canada; ${ }^{3}$ Department of Medical Imaging, University Health Network, University of Toronto, Toronto, ON, Canada; ${ }^{4}$ Department of Pathology, University Health Network, University of Toronto, Toronto, ON, Canada

Cite as: Can Urol Assoc J 2017;11 (1-2):41-6. htrtp://dx.doi.org/10.5489/cuaj.4031

\section{Abstract}

Introduction: We sought to determine if prostate cancer (PCa) is associated with worse lower urinary tract symptoms (LUTS) than matched benign prostates, with attention to cancer characteristics, in a contemporary cohort.

Methods: Using a single-institution database (January 1, 2009June 30, 2013), men diagnosed with PCa on biopsy and controls with negative biopsies were matched 1:1 on age, prostate volume, and a propensity score predicting the probability of PCa diagnosis. International Prostate Symptom Score (IPSS) was compared between PCa cases and controls using paired statistics, stratifying on grade, cancer volume, stage, and D'Amico risk group. Sensitivity analyses were performed separately, repeating the match for highgrade, high-volume, and high-stage cancers only, and excluding users of benign prostatic hyperplasia medications.

Results: In our cohort of 1330 men (665 with PCa), there were 284 (42.7\%) Gleason 6 cancers (Grade Group 1), 315 (47.4\%) Gleason 7 cancers (Grade Group 2-3), and 66 (9.9\%) Gleason 8-10 cancers (Grade Group 4-5). There was no difference in IPSS between PCa cases (median 6.5, interquartile range [IQR] 3-12) and benign controls (median 7, IQR 3-13; $\mathrm{p}=0.34$ ). Subgroup analyses based on cancer grade, volume, or stage, showed no significant differences in IPSS between men with and without PCa, except among men with cT2b-cT4 PC (median 9, IQR 5-16) vs. matched benign counterparts (median 8, IQR 3-12; $\mathrm{p}=0.03$ ). Sensitivity analyses supported these findings.

Conclusions: Modern PCa does not appear to be associated with worse LUTS compared to benign prostates of the same size. Outlet obstruction is likely a late event in the natural history of PCa. This has implications for timely PCa detection, which should ideally be prior to the onset of LUTS.

\section{Introduction}

Lower urinary tract symptoms (LUTS) secondary to benign prostatic hyperplasia $(\mathrm{BPH})$ affect more than $60 \%$ of men over the age of $60 .^{1-4}$

Prostate cancer (PCa) is often considered in the differential diagnosis of LUTS, based on conventional experience and cross-sectional data from the pre-prostate-specific antigen (PSA) era. ${ }^{5-7}$ However, cancers being diagnosed in the modern era are very different from the pre-PSA era. Evidence substantiating whether PCa contributes to LUTS in the contemporary setting is lacking.

Thus, we sought to determine if a diagnosis of PCa is associated with worse LUTS compared to matched controls without PCa, in a contemporary cohort. Additionally, in order to understand the characteristics of PCa that may result in LUTS, the aim was also to determine if the association varies by D'Amico risk group at diagnosis, cancer volume on biopsy, grade, and clinical stage. We hypothesized that only men with high D'Amico risk PCa, high-volume, and high-stage cancers would have worse LUTS compared to matched controls.

\section{Methods}

\section{Patients and data collection}

Patients undergoing biopsy from January 1, 2009-June 30, 2013 were identified using our prospectively maintained institutional database of men referred for transrectal ultrasound (TRUS)-guided biopsy for PCa suspicion..$^{8,9}$ Men with previous transurethral resection of the prostate (TURP) were excluded. For patients with multiple biopsies, the most recent was used. Institutional research ethics board approval 
Bhindi et al.

was obtained, and patient consent was sought for inclusion in the database.

Patient questionnaires were completed to ascertain ethnicity, family history of PCa, and use of $5 \alpha$-reductase inhibitors (5-ARI) or alpha-blockers. Electronic patient charts were reviewed to gather the patient's digital rectal exam (DRE) findings, TRUS-measured prostate volume (TPV), and PSA values. Biopsies were performed by two high-volume radiologists. Initial biopsies generally involved 10-12 cores and repeat biopsies involved 13-18 cores. Additional cores were taken if suspicious lesions were seen on TRUS. Biopsies were read by genitourinary pathologists.

\section{Exposure and outcome}

PCa was classified by cancer volume on biopsy, Gleason grade, clinical stage (American Joint Committee on Cancer tumor-node-metastasis [AJCC TNM] staging, seventh edition). ${ }^{10}$ Low-volume cancer was defined as $\leq 3$ cores or $\leq 1 / 3$ of total number of cores involved, and no core with $>50 \%$ cancer involvement, based on institutional criteria for low-volume cancer for active surveillance. ${ }^{11} \mathrm{High}$-volume cancer was arbitrarily defined a priori as $>50 \%$ of cores involved and $>50 \%$ cancer involvement in at least one core. Intermediate-volume was defined as all other cancers not fulfilling criteria for low- or high-volume. Patients were also grouped according to the D'Amico classification into low(Gleason 6 [Grade Group 1], stage cT1c/cT2a, and PSA $<10 \mathrm{ng} / \mathrm{ml}$ ), intermediate- (Gleason 7 [Grade Group 2-3], stage cT2b/cT2c or PSA 10-20ng/ml); and high-risk (Gleason 8-10 [Grade Group 4-5], stage cT3/cT4 or PSA >20ng/ml).

The primary outcome was LUTS, as assessed using an International Prostate Symptom Score (IPSS) questionnaire ${ }^{12,13}$ administered immediately prior to biopsy.

\section{Matching}

A propensity score to predict the probability of PCa diagnosis was created based on age, prostate volume, ethnicity, family history of $\mathrm{PCa}$, prior negative biopsy, alpha-blocker, and 5-ARI use. In order to avoid introducing bias by artificially forcing balance, abnormal DRE or serum PSA were not included in the propensity score (i.e., patients with and without PCa should not be balanced on DRE and PSA). Patients with and without PCa were then hard-matched on age, prostate volume, and logit of propensity score.

\section{Statistical analysis}

Statistical analyses were performed using SAS 9.3 (SAS Institute Inc., Cary, NC, U.S.). All tests were two-sided, with $\mathrm{p} \leq 0.05$ considered statistically significant. Standardized differences were used to assess balance of clinical characteris- tics between men with and without PCa. The non-parametric Wilcoxon signed rank test and box-and-whisker plots were used to compare IPSS scores between men with and without PCa since values did not follow a normal distribution. In order to evaluate how cancer volume, grade, and stage influenced the association between PCa and LUTS, subgroup analyses were performed stratifying by these parameters (volume: low vs. intermediate vs. high, as previously defined; grade: Gleason 6 vs. 7 vs. 8-10; clinical stage: cT1c/T2a vs. cT2b-T4; D'Amico risk classification: low vs. intermediate vs. high).

The number of African-Canadians was somewhat imbalanced in our main matched cohort and this could not be corrected by altering the calipers of the propensity score. We therefore performed a sensitivity analysis that repeated the match while excluding these subjects to determine if this was influencing our results. We also performed sensitivity analyses assessing whether $\mathrm{BPH}$ treatment was introducing bias in the subgroup analyses evaluating high-volume cancer on biopsy, high-grade, and high-stage disease. In order to maximize the number of patients matched for each analysis, the match was separately repeated looking at each of the following groups: 1) high-volume cancers; 2 ) high-grade cancers (Gleason 8-10); 3) high clinical T-stage cancers (clinical T2b-T4). In each of these matched sensitivity analyses, patients on medical therapy for BPH (alpha blockers or 5-ARIs) were excluded.

\section{Results}

There were 2055 (997 with PCa) eligible men in the database. The final matched cohort included 1330 men (665 with $\mathrm{PCa}$ ). Based on this sample size, our study had a power of $90 \%$ to detect a difference in median IPSS score of 1.1 between groups (with alpha $=0.05$ ).

Study groups were balanced for all matched parameters (standardized difference $\leq 0.10$ ), with exception to AfricanCanadian ethnicity (standardized difference $=0.27$; Table 1 ). Mean age of the total cohort was 62.5 years (standard deviation [SD] 7.4), and median TPV was $42 \mathrm{~mL}$ (interquartile range [IQR] 32-53 mL). Alpha blockers and 5-ARIs were being used by $170(12.8 \%)$ and $134(10.0 \%)$ men, respectively. Table 2 summarizes the cancer-related characteristics of the PCa group.

In the primary analysis (Fig. 1), there was no significant difference in IPSS score when comparing men with PCa (median 7, IQR 3-12) vs. men without PCa on biopsy (median 7, IQR 3-13; $p=0.34$ ). When stratified by D'Amico risk group (Fig. 2), there were no significant differences between men with low- $(p=0.25)$, intermediate- $(p=0.42)$ or high-risk $(p=0.46)$ PCa vs. matched controls.

The remainder of the subgroup analyses are illustrated in Supplementary Fig. 1. There was no significant differ- 


\begin{tabular}{|c|c|c|c|}
\hline $\begin{array}{l}\text { Patient } \\
\text { characteristics }\end{array}$ & $\begin{array}{c}\mathrm{PCa} \\
\mathrm{n}=665\end{array}$ & $\begin{array}{c}\text { No PCa } \\
n=665\end{array}$ & $\begin{array}{c}\text { Standardized } \\
\text { difference }\end{array}$ \\
\hline $\begin{array}{l}\text { Age in years, mean } \\
\text { (SD) }\end{array}$ & $62.4(7.5)$ & $62.3(7.6)$ & 0.01 \\
\hline $\begin{array}{l}\text { Prostate volume in } \\
\mathrm{mL} \text {, median (IQR) }\end{array}$ & $42(31-53)$ & $42(32-53)$ & 0.02 \\
\hline \multicolumn{4}{|l|}{ Ethnicity } \\
\hline Caucasian & $463(69.6)$ & 478 (71.9) & 0.05 \\
\hline African descent & $63(9.5)$ & $20(3.0)$ & 0.27 \\
\hline Mixed/other & $139(20.9)$ & $167(25.1)$ & 0.10 \\
\hline $\begin{array}{l}\text { Known family } \\
\text { history of PCa, } \mathrm{n}(\%)\end{array}$ & $147(22.1)$ & $141(21.2)$ & 0.02 \\
\hline $\begin{array}{l}\text { History of prior } \\
\text { biopsy, } \mathrm{n}(\%)\end{array}$ & $153(23.0)$ & $153(23.0)$ & 0.00 \\
\hline $\begin{array}{l}\text { Use of alpha } \\
\text { blocker, n (\%) }\end{array}$ & $83(12.5)$ & $87(13.1)$ & 0.02 \\
\hline Use of 5-ARI, n (\%) & $66(9.9)$ & $68(10.2)$ & 0.01 \\
\hline $\begin{array}{l}\text { Abnormal DRE, } n \\
(\%)\end{array}$ & $200(30.1)$ & $149(22.4)$ & 0.17 \\
\hline $\begin{array}{l}\text { Serum PSA in ng/ } \\
\text { ml, median (IQR) }\end{array}$ & $\begin{array}{c}6.10 \\
(4.49-8.76) \\
\end{array}$ & $\begin{array}{c}5.20 \\
(3.51-7.00) \\
\end{array}$ & 0.23 \\
\hline
\end{tabular}

ence in IPSS between men with low-volume $(p=0.39)$, intermediate-volume $(p=0.12)$, and high-volume $(p=0.25)$ cancer on biopsy compared to matched controls. Similarly, when stratified by grade, median IPSS scores were not significantly different for men with Gleason 6/Grade Group $1(p=0.26)$, Gleason 7/Grade Group 2-3 ( $p=0.53)$, and Gleason 8-10/ Grade Group 4-5 ( $p=0.48)$ cancers compared to matched controls. When stratified by clinical stage, the IPSS scores of men with clinical T1c/T2a PCa were not significantly different from matched controls $(p=0.09)$. Conversely, the IPSS scores of men with clinical T2b-T4 cancers (median 9, IQR 5-16) were significantly higher than matched controls (median 8, IQR 3-12; $\mathrm{p}=0.03$ ).

To assess whether the imbalance of patients with AfricanCanadian ethnicity between study arms influenced study findings, our first sensitivity analysis repeated the match, excluding these patients. These results were similar to the main analysis, and no significant difference in IPSS was noted between men with and without PCa ( $p=0.63$; Supplementary Fig. 2). In the second set of sensitivity analyses, we repeated separate matches for men with high-volume cancer on biopsy, high-grade $\mathrm{PCa}$, and high-stage $\mathrm{PCa}$, all while excluding men using $\mathrm{BPH}$ medications; none of the comparisons reached statistical significance (Supplementary Fig. 2).

\section{Discussion}

The comparison of LUTS between men with and without PCa on biopsy is challenging. The absence of LUTS is a predictive risk factor for cancer detection on biopsy among

\begin{tabular}{|c|c|c|}
\hline Prostate cancer variables & & $\begin{array}{c}\text { Total } \\
\text { PCa arm } \\
\text { (n=665) }\end{array}$ \\
\hline \multirow[t]{5}{*}{ Clinical T-stage, n (\%) } & T1c & 465 (69.9) \\
\hline & $\mathrm{T} 2 \mathrm{a}$ & $137(20.6)$ \\
\hline & $\mathrm{T} 2 \mathrm{~b}$ & $16(2.4)$ \\
\hline & $\mathrm{T} 2 \mathrm{c}$ & $25(3.8)$ \\
\hline & T3-4 & $22(3.3)$ \\
\hline \multirow[t]{3}{*}{ Gleason score, n (\%) } & 6 (Grade Group 1) & $284(42.7)$ \\
\hline & $\begin{array}{c}7 \text { (Grade Group } \\
2-3 \text { ) }\end{array}$ & $315(47.4)$ \\
\hline & $\begin{array}{l}\text { 8-10 (Grade } \\
\text { Group 4-5) }\end{array}$ & $66(9.9)$ \\
\hline \multirow[t]{3}{*}{ Cancer volume on biopsy, $\mathrm{n}(\%)$} & Low & $374(56.2)$ \\
\hline & Intermediate & $177(26.6)$ \\
\hline & High & $114(17.1)$ \\
\hline \multirow[t]{3}{*}{ D'Amico risk group, $\mathrm{n}(\%)$} & Low & $253(38.0)$ \\
\hline & Intermediate & $306(46.0)$ \\
\hline & High & $106(18.0)$ \\
\hline \multicolumn{2}{|c|}{ Positive cores (\% of total), median (IQR) } & $25(11-45)$ \\
\hline \multicolumn{2}{|c|}{ Max core \% involvement, median (IQR) } & $25(10-60)$ \\
\hline
\end{tabular}

patients referred for an elevated PSA. ${ }^{14}$ Meanwhile, patients with a negative biopsy are more likely to have BPH (i.e., larger prostates and worse LUTS), explaining the elevated PSA that prompted their initial biopsy referral. ${ }^{15,16}$ Therefore, by direct comparison, patients with PCa may appear to have lower IPSS scores. Our study used propensity score-matching to address this source of confounding by inducing balance in the prevalence of $\mathrm{BPH}$ between groups. Notably, in our matched cohort, men with and without PCa had virtually identical distributions of prostate volume and similar rates of BPH medication usage.

In our study, there was no significant difference in IPSS scores between men with and without PCa on biopsy. Given that our analysis was well-powered to detect a clinically relevant difference, type II error is unlikely. This suggests that LUTS in contemporary patients with PCa are more likely due to concurrent $\mathrm{BPH}$ rather than their cancer., ${ }^{3,17}$

Given that approximately $70 \%$ of PCa originates in the peripheral zone, ${ }^{1,18}$ where early cancers would seldom exert a mass effect on the prostatic urethra, subgroup analyses were performed to explore whether more aggressive or locally advanced disease would have worse LUTS compared to matched controls. Patients with higher D'Amico risk, highergrade $\mathrm{PC}$, and higher cancer volume on biopsy did not have worse IPSS scores compared to matched controls. While differences in IPSS between patients with cT2b-T4 disease and matched controls reached statistical significance (median 9,

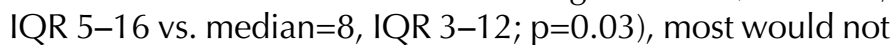
consider this difference clinically significant. For example, 
Bhindi et al.

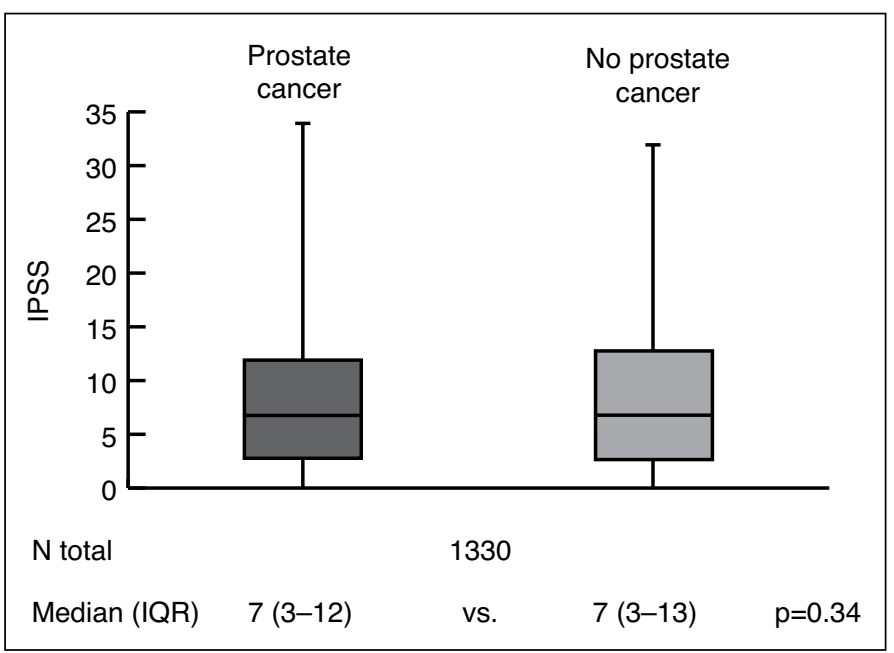

Fig. 1. Comparison of International Prostate Symptom Score (IPSS) between men with and without prostate cancer: Whole cohort analysis. IQR: interquartile range.

a change in IPSS of at least 3-4 points corresponds to a clinically detectable change in patients' global feeling of urination. ${ }^{19,20}$

Our findings likely suggest that the development LUTS is a late event in the natural history of PCa. Peripheral zone PCa identified at the symptomatic stage is often more advanced and locally spread, and therefore less amenable to cure. ${ }^{5,7}$ Ductal PCa is a rare exception, since it originates centrally near the prostatic ducts and can cause bladder outlet obstruction without having to spread beyond the transition zone. ${ }^{21}$

Our findings have implications for PSA screening, which has been scrutinized over concerns regarding PCa overdiagnosis and overtreatment. ${ }^{22,23}$ However, if PSA screening in asymptomatic men is abandoned entirely, and we instead rely on the development of symptoms to prompt work up for $\mathrm{PCa}$, we truly risk passing up on the opportunity for cure. ${ }^{24,25}$

Our findings also have implications for the routine evaluation for LUTS. Men presenting to their primary care physician with LUTS, particularly those with a benign DRE, are unlikely to find $\mathrm{PCa}$ at the root of their symptoms. ${ }^{17}$ In such patients, PSA elevation related to $\mathrm{BPH}$ is a common finding and can trigger further evaluation for $\mathrm{PCa}$, independent from their presenting complaint. A recent study showed that men over the age of 65 undergoing treatment for LUTS, compared to untreated men, were over twice as likely to undergo a prostate biopsy during a 10-year followup, but were no more likely to be diagnosed with PCa. ${ }^{26}$ In an era where serum PSA testing is being subjected to increasing scrutiny, in the absence of an abnormal DRE, the role of a diagnostic PSA test as part of the routine evaluation of LUTS needs to be revisited, ${ }^{27,28}$ particularly among older men who may be harmed by overdiagnosis. This is an issue that is separate from using PSA as a screening test in men with sufficient life expectancy who would benefit from the early detection of PCa.

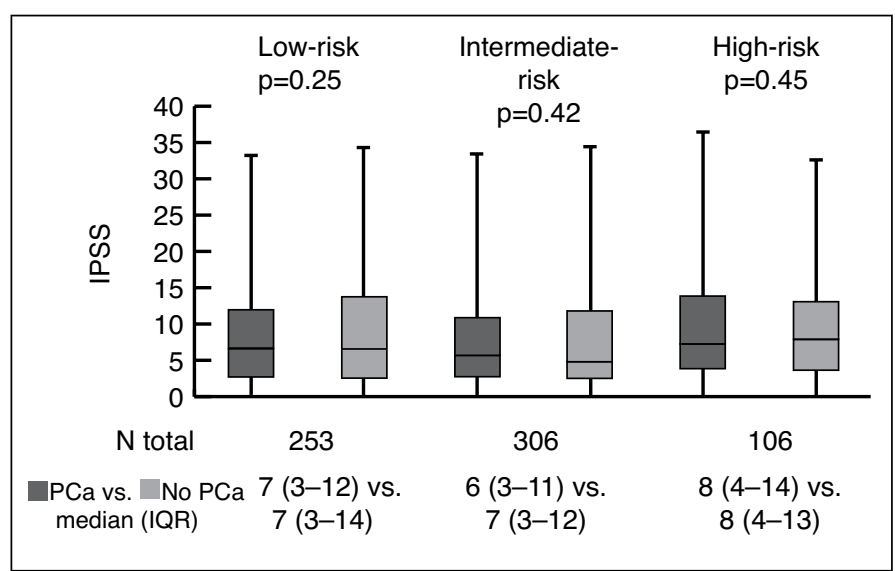

Fig. 2. Comparison of International Prostate Symptom Score (IPSS) between men with and without prostate cancer: Subgroup analyses by D'Amico risk group. IQR: interquartile range; PCa: prostate cancer.

The strengths of this study include its methodological rigour to control for potential confounders. To the best of our knowledge, our report is the largest to evaluate the association between PCa and LUTS in a contemporary cohort. It is also the largest contemporary study to evaluate the extent to which D'Amico risk group, cancer volume, grade, and clinical stage play into the development of LUTS.

There are limitations to this study. Due to the low number of patients with clinical stages T2b or greater, we were required to pool all such patients together for statistical purposes. Our study was not adequately powered to perform subgroup analyses comparing T2b-c, T3, and T4 clinical stages. Therefore, apart from suggesting that the onset of LUTS is a relatively late event in the local progression of PCa, our data cannot assess the T-stage at which LUTS begin to develop. Finally, our control group may be different than the general population of healthy men without PCa in unmeasurable ways. To the best of our abilities within an observational study design, we accounted for several measurable parameters, including prostate volume and $\mathrm{BPH}$ medication use.

\section{Conclusion}

In our contemporary cohort that was well-powered to detect an IPSS change of one point, PCa was not associated with worse LUTS compared to controls with a negative biopsy. Bladder outlet obstruction from cancer is likely a late event in the natural history of PCa. Therefore, in an era where $\mathrm{PCa}$ screening is undergoing increasing scrutiny, the role of PSA testing in the routine diagnostic evaluation for male LUTS needs to be revisited, since an extensive search for PCa in this population will likely lead to PCa overdiagnosis. Conversely, PSA screening in asymptomatic men should not be abandoned, since if we rely on the onset of LUTS to prompt a workup for men harbouring PCa, then we will truly 
risk missing the opportunity for cure in a large number of men. The pros and cons of PSA testing should be discussed in all instances.

Competing interests: Dr. Hamilton was the principal investigator on the SPARTAN trial and a consultant/lecturer for AbbVie and Bayer. Dr. Fleshner has been a consultant for Amgen, Astellas, and Janssen; has participated in clinical trials for Amgen, Astellas, Ferring, and Janssen; and has received grant funding from Canadian Cancer Society Research Institute and Prostate Cancer Canada. The remaining authors report no competing personal or financial interests.

This paper has been peer-reviewed.

\section{References}

1. Alcaraz A, Hammerer $\mathrm{P}$, Tubaro $\mathrm{A}$, et al. Is there evidence of a relationship between benign prostatic hyperplasia and prostate cancer? Findings of a literature review. Eur Urol 2009;55:864-73. https://doi.org/10.1016/i.eururo.2008.11.011

2. Irwin DE, Milsom I, Hunskaar $S$, et al. Population-based survey of urinary incontinence, overactive bladder, and other lower urinary tract symptoms in five countries: Results of the EPIC study. Eur Urol 2006;50:130614; discussion 14-5. https://doi.org/10.1016/i.eururo.2006.09.019

3. Gades NM, Jacobson DJ, Girman CJ, et al. Prevalence of conditions potentially associated with lower urinary tract symptoms in men. BJU Int 2005;95:549-53. https://doi.org/10.1111/i.1464410X.2005.05337.x

4. Abrams P, Chapple C, Khoury S, et al. International Scientific Committee. Evaluation and treatment of lower urinary tract symptoms in older men. J Urol 2009;181:1779-87. https://doi.org/10.1016/i. juro.2008.11.127

5. Levy IG, Gibbons L, Collins JP, et al. Prostate cancer trends in Canada: Rising incidence or increased detection? Can Med Assoc J 1993;149:617-24.

6. Corder EH, Chute CG, Guess HA, et al. Prostate cancer in Rochester, Minnesota (USA), from 1935 to 1989: Increases in incidence related to more complete ascertainment. Cancer Causes Control 1994;5:207-14. https://doi.org/10.1007/BF01830238

7. McClelland JC. The early diagnosis of cancer in the bladder, prostate, and kidney. Can Med Assoc J 1934;31:165-7.

8. Bhindi B, Locke J, Alibhai SM, et al. Dissecting the association between metabolic syndrome and prostate cancer risk: Analysis of a large clinical cohort. Eur Urol 2015;67:64-70. https://doi.org/10.1016/i. eururo.2014.01.040

9. Bhindi B, Mamdani M, Kulkarni GS, et al. Impact of the U.S. Preventive Services Task Force recommendations against prostate specific antigen screening on prostate biopsy and cancer detection rates. J Urol 2015;193:1519-24. https://doi.org/10.1016/i.juro.2014.11.096

10. Edge SB, Compton CC. The American Joint Committee on Cancer: The 7th edition of the AJCC cancer staging manual and the future of TNM. Ann Surg Oncol 2010;17:1471-4. https://doi.org/10.1245/ s10434-010-0985-4

11. Bhindi B, Kulkarni GS, Finelli A, et al. Obesity is associated with risk of progression for low-risk prostate cancers managed expectantly. Eur Urol 2014;66:841-8. https://doi.org/10.1016/i.eururo.2014.06.005

12. Barry MJ, Fowler FJ Jr, $O^{\prime}$ Leary MP, et al. The American Urological Association symptom index for benign prostatic hyperplasia. The Measurement Committee of the American Urological Association. I Urol 1992;148:1549-57; discussion 64
13. Glasser DB, Carson C 3rd, Kang JH, et al. Prevalence of storage and voiding symptoms among men aged 40 years and older in a US population-based study: Results from the Male Attitudes Regarding Sexual Health study. Int I Clin Pract 2007;61:1294-300. https://doi.org/10.1111/i.1742-1241.2007.01454.x

14. Porter CR, Kim J. Low AUA symptom score independently predicts positive prostate needle biopsy: Results from a racially diverse series of 411 patients. Urology 2004;63:90-4. https://doi.org/10.1016/i. urology.2003.08.019

15. Meigs JB, Barry MJ, Oesterling JE, et al. Interpreting results of prostate-specific antigen testing for early detection of prostate cancer. J Gen Intern Med 1996;11:505-12. https://doi.org/10.1007/ BF02599596

16. Hochberg DA, Armenakas NA, Fracchia JA. Relationship of prostate-specific antigen and prostate volume in patients with biopsy proven benign prostatic hyperplasia. Prostate 2000;45:315-9. https://doi.org/10.1002/1097-0045(20001201)45:4<315::AID-PROS5>3.0.C0;2-2

17. Schenk JM, Kristal AR, Arnold KB, et al. Association of symptomatic benign prostatic hyperplasia and prostate cancer: Results from the prostate cancer prevention trial. Am J Epidemiol 2011;173:1419-28. https://doi.org/10.1093/aje/kwq493

18. McNeal JE, Villers AA, Redwine EA, et al. Capsular penetration in prostate cancer. Significance for natural history and treatment. Am J Surg Pathol 1990;14:240-7. https://doi.org/10.1097/00000478199003000-00005

19. Barry MJ, Cantor A, Roehrborn CG, et al. Relationships among participant international prostate symptom score, benign prostatic hyperplasia impact index changes, and global ratings of change in a trial of phytotherapy in men with lower urinary tract symptoms. J Urol 2013;189:987-92. https://doi.org/10.1016/i.juro.2012.08.257

20. Barry MJ, Williford WO, Chang Y, et al. Benign prostatic hyperplasia specific health status measures in clinical research: How much change in the American Urological Association symptom index and the benign prostatic hyperplasia impact index is perceptible to patients? J Urol 1995;154:1770-4. https://doi.org/10.1016/S0022-5347(01)66780-6

21. Meeks JJ, Zhao LC, Cashy J, et al. Incidence and outcomes of ductal carcinoma of the prostate in the USA: Analysis of data from the Surveillance, Epidemiology, and End Results program. BJU Int 2012;109:831-4. https://doi.org/10.1111/i.1464-410X.2011.10520.x

22. Moyer VA, Force USPST. Screening for prostate cancer: U.S. Preventive Services Task Force recommendation statement. Ann Intern Med 2012;157:120-34. https://doi.org/10.7326/0003-4819-157-2201207170-00459

23. Chou R, Croswell JM, Dana T, et al. Screening for prostate cancer: A review of the evidence for the U.S. Preventive Services Task Force. Ann Intern Med 2011;155:762-71. https://doi.org/10.7326/00034819-155-11-201112060-00375

24. Kilpelainen TP, Auvinen A, Maattanen L, et al. Results of the three rounds of the Finnish Prostate Cancer Screening Trial — the incidence of advanced cancer is decreased by screening. Int I Cancer 2010;127:1699-705. https://doi.org/10.1002/iic.25368

25. Mahmood U, Levy LB, Nguyen PL, et al. Current clinical presentation and treatment of localized prostate cancer in the United States. J Urol 2014;192:1650-6. https://doi.org/10.1016/i.juro.2014.06.017

26. Weight CJ, Kim SP, Jacobson DJ, et al. The effect of benign lower urinary tract symptoms on subsequent prostate cancer testing and diagnosis. Eur Urol 2013;63:1021-7. https://doi.org/10.1016/i. eururo.2012.12.060

27. Abrams $P$, Chapple $C$, Khoury $S$, et al. Evaluation and treatment of lower urinary tract symptoms in older men. J Urol 2013;189:S93-101. https://doi.org/10.1016/i.juro.2012.11.021

28. Heidenreich A, Bastian PJ, Bellmunt J, et al. EAU guidelines on prostate cancer. Part 1: Screening, diagnosis, and local treatment with curative intent-update 2013. Eur Urol 2014;65:124-37. https://doi.org/10.1016/j.eururo.2013.09.046

Correspondence: Dr. Bimal Bhindi, Division of Urology, Department of Surgery, University Health Network, University of Toronto, Toronto, ON, Canada; bimal.bhindi@mail.utoronto.ca 
Bhindi et al.

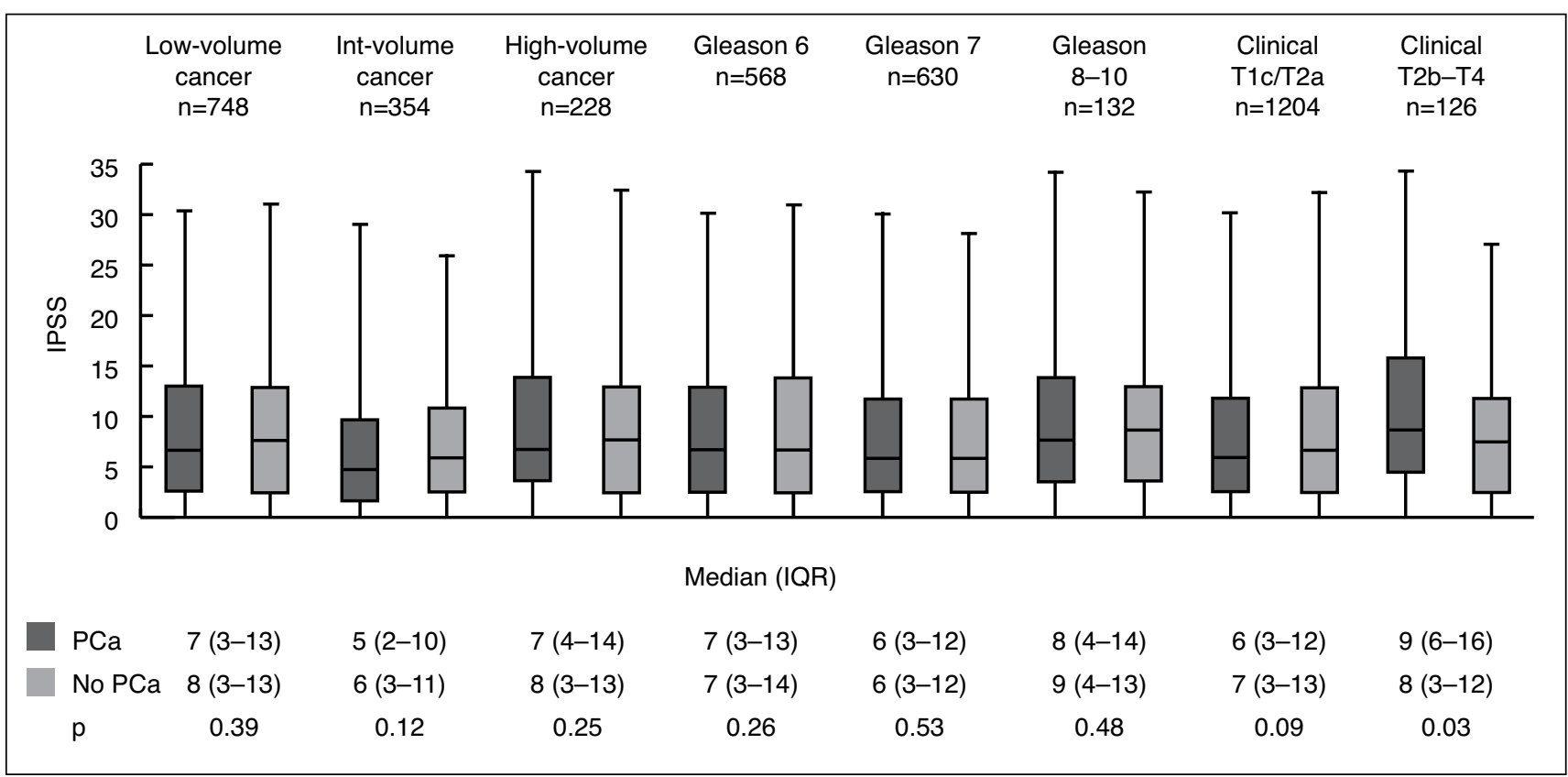

Supplementary Fig. 1. Subgroup analyses by cancer volume, grade, and stage. IPSS: International Prostate Symptom Score; IQR: interquartile range; PCa: prostate cancer.

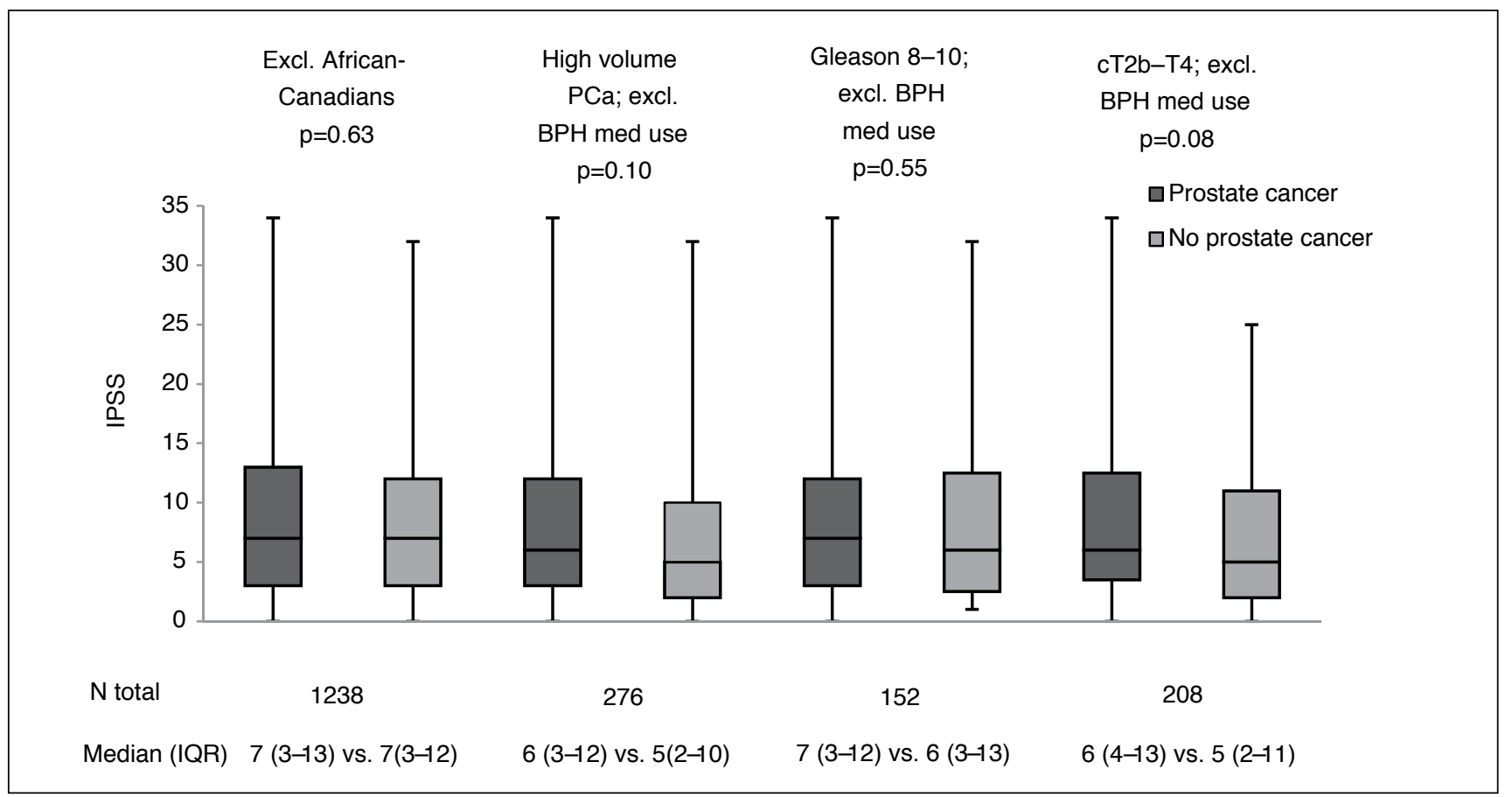

Supplementary Fig 2. Sensitivity analyses comparing International Prostate Symptom Score (IPSS) between matched subjects with and without prostate cancer (PCa). BPH: benign prostatic hyperplasia; IQR: interquartile range. 